\title{
AUDITOR'S REPORT
}

January 29, 1976

Board of Directors

African Studies Association, Inc.

Members of the Board:

I have examined the Balance Sheet of the African Studies Association, Inc. at December 31, 1975 and the Statement of Changes in Fund Balances and Statement of Revenues and Disbursements for the year then ended. My examination was made in accordance with generally accepted auditing standards and accordingly included such tests of the accounting records and such other auditing procedures as $I$ considered necessary in the circumstances.

In my opinion, the accompanying Balance Sheet, Changes in Fund Balances, and Statement of Revenues and Disbursements present fairly the financial position of African Studies Association, Inc. at December 31, 1975 and the results of its operations for the year then ended, in conformity with generally accepted principles of accounting applied on a basis consistent with that of the preceding year except for investment securities. In prior years they were shown at cost; at December 31, 1975 they are shown at market value with the reduction also applied to the General Fund Balance. This change in presentation has no effect on operations for the year 1975.

Yours very truly,

Dr. William C. Lins

Certified Public Accountant

\section{EXHIBIT "A"-Balance Sheet}

\section{Assets}

Current Assets:

Cash in bank

Assets Held in Agency Account:

Securities at cost (approximate market value,

December 31,1975 is $\$ 43,450$ )

Less allowance to reduce securities to market

Uninvested cash

$\$ 62,262$

18,812

43,450

669

44,119

Fixed Assets:

Office equipment

Less accumulated depreciation

29,134

4,390

Total Assets

\section{$\$ 72,266$}

\section{Outside Liabilities and Fund Balance}

Liabilities:

Accrued disbursements

$\$ 26,984$

5,100

Accounts payable

Payroll taxes and withhold payable

14,477

Membership dues received in advance

Total Liabilities

47,349

General Fund Balance (Exhibit "B")

24,917

Total Outside Liabilities and Fund Balance 
Balances, January 1, 1975

Revenues less disbursements-Year 1975

Transfer from RLC to General Fund

Less allowance required to reduce carrying value of securities from cost to market

Balance, December 31, 1975

\section{EXHIBIT "C"-Statement of Revenues and Disbursements}

\section{Revenues:}

Advertising

Annual meeting gross receipts:

Registrations

Exhibits and advertising

Grants from The Ford Foundation

Income from investments

Interest on savings account

Membership dues

Rental fees-mailing list

Sales of publications

Sundry

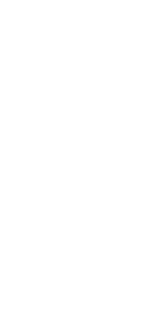

Total Revenues

$\$$

38,975

62,573

32,771

155,613

\section{Disbursements:}

Accounting and bookkeeping

Agency fee

Annual meeting

Board expenses

Committees:

Current issues

Herskovits Award

Publications

Women

Computer Services

Depreciation of office equipment

Donations

Duplication and printing

General administration

Grants for research

Herskovits award

Health insurance

History in Africa

Insurance

Issue

Mailing list maintenance

Meetings and conferences

Newsletter

Office supplies

Payroll taxes

Periodicals and publications

Postage

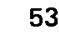

535

535

$\$$

7,666

7,267

1,837

144

3,573

272

7,666

7,267

5,000

1,837

144

62,573

3,573

32,771

272

121,638

33,975

Research

Liaison Fund

$\$(7,485)$

18,265

10,780

$(10,780)$

33,975

$\begin{array}{rr}3,338 & 3,338 \\ 801 & 801 \\ 4,649 & 4,649 \\ 8,087 & 8,087\end{array}$

547

547

31

695

31

695

500

$2,384 \quad 1,396$

$2,254 \quad 2,254$

$1,004 \quad 1,004$

$8,359 \quad 7,787$

28,726

572

30,309

6,850

500

808

3,564

537

9,735

45

330

4,685

3,964

1,975

242

6,156

6,156

$-6$. 
Publications

Repairs and maintenance of office equipment

Return of unused portion of grant from

The Ford Foundation

Review

Review of Books

Salaries

Sundry

Telephone and telegraph

Travel

Total Disbursements

Revenues less Disbursements

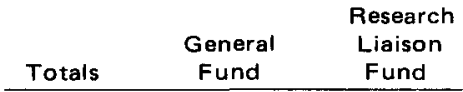

\begin{tabular}{|c|c|c|}
\hline $\begin{array}{r}7.174 \\
206\end{array}$ & $\begin{array}{r}2,088 \\
206\end{array}$ & 5,086 \\
\hline 5,000 & 5,000 & \\
\hline 6,093 & 6,093 & \\
\hline 2,061 & 2,061 & \\
\hline 32,764 & 32,764 & \\
\hline 340 & 340 & \\
\hline 1,897 & 1,508 & 389 \\
\hline 169 & 169 & \\
\hline 158,053 & 142,343 & 15,710 \\
\hline$(2,440)$ & $\$(20,705)$ & $\$ 18,265$ \\
\hline
\end{tabular}

\section{MEETINGS-PAST \& FUTURE}

Western Association of Africanists Annual Meeting. Colorado State University, Fort Collins, Colorado. (April 1-3, 1976). Write: Professor J. Leo Cefkin, Program Chairman, Western Association of Africanists, Colorado State University, Fort Collins, Colorado.

University of California Great Spring Palaver. University of California, Santa Barbara, California. (April 16-17, 1976). Theme: The African Genius. For further information, contact: UCSB African Studies Committee, c/o Black Studies Department and African Area Studies, 4631 South Hall, University of California, Santa Barbara, California 93106.

Africana Festival 1976. National Historical Museum, Stockholm, Sweden. (April 21-May 2, 1976). Festival will focus on African history, politics, literature, and the arts since 1945. Write: The Committee for Africana Festival, Essinge Brogata 7/Ogebratt, 11261 Stockholm, Sweden.

The African Heritage Studies Association Eighth Annual Meeting. Sheraton Biltmore Hotel, Atlanta, Georgia. (April 22-25, 1976). Theme: A Luta Continua? Revolution and Counter Revolution in the African World. Contact: Joan E. Wilson, Chairperson, Division of Social Sciences, Atlanta Junior College 1630 Stewart Avenue, S.W., Atlanta, Georgia 30315, tel. (404) 656-6368 or Edward Fontenette, Associate Dean, Library, Atlanta University, 233 Chestnut Street, N.W. Atlanta, Georgia 30314, tel. (404) 681-0251.

Symposium on the African Dispersal: Expectations and Realities. George Sherman Union, Boston University, Boston, Massachusetts. (April 24, 1976, 9:30-5:00). Papers by: Elliott Skinner; Emilia da Costa; Joseph Harris; Edward Brathwaite; Wilson Head. Contact: Boston University Afro-American Studies Center, 138 Montfort Street, Boston, Massachusetts 02146.

DRODAT (Drought Data): A Planning Workshop. Environmental Training Program, Dakar, Senegal. (April 28-May 3, 1976). The workshop is jointly sponsored by ENDA, The Institut de Recherche en Sciences Humaines, Niamey, Clark University, Worcester, Massachusetts, and the International Development Research Centre, Canada. Participants will plan cooperative research efforts to retrieve information about the recent Sahelian drought. A detailed report of the planning workshop should be available about June 1, 1976. Anyone interested in receiving a copy of the report (indicate French or English version) should write either: DRODAT ENDA, BP 3370, Dakar, Senegal, or DRODAT, Program of International Development, Clark University, Worcester, Massachusetts 01610.

Third Annual Conference of the New York African Studies Association (NYASA). Cornell University, Ithaca, New York. (April 30-May 1, 1976). Write: J. Congress Mbata, Conference Chairman, Africana Studies and Research Center, Cornell University 310 Triphammer Road, Ithaca, New York 14850. 